\title{
An SEM-based X-ray Microtomography System
}

\author{
P.R. Miller*, S.C. Mayo*, T. Gureyev*, S.W. Wilkins*, J. Sheffield-Parker** \\ * CSIRO Manufacturing and Infrastructure Technology, PB 33, Clayton South, VIC 3169, Australia \\ ** XRT Limited, Suite A.3.1, 63 Turner Street, Port Melbourne, VIC 3207, Australia
}

The X-ray ultraMicroscope $(\mathrm{XuM})$ is an SEM-based projection X-ray microscope that allows X-ray images to be recorded with resolution better than $100 \mathrm{~nm}$ [1-3]. The projection method for X-ray microscopy is very simple in principle: X-rays from the source pass through the sample to form a projected image at the detector and magnification is varied by moving the sample between the source and the detector. The sample may be rotated to record a tomographic data series.

A consequence of the significant separation between the sample and the detector, coupled with the small X-ray source size, is that the image will show both in-line phase-contrast as well as absorption contrast. XuM images exploit both absorption contrast and phase contrast to reveal fine internal structure and edge detail. Phase retrieval algorithms can be used to extract quantitative data from $\mathrm{XuM}$ images, to improve image quality and to aid interpretation, and to transform the images into a form more suitable for tomographic processing.

The procedure for acquiring and processing tomographic data is as follows:

1. The sample is rotated about the feature of interest to record the tomographic data series. Eucentric rotation is used; the feature does not have to lie on the stage rotation axis.

2. If necessary, blurring due to the asymmetric extended X-ray source is removed by deconvolution using a model for the source calculated using the Monte Carlo method.

3. Phase retrieval is applied to the image series.

4. Images are registered to correct for drift and positioning errors.

5. Sinograms are prepared.

6. The FDK cone-beam algorithm [4] is used to reconstruct slices through the sample.

7. Commercial $3 \mathrm{D}$ rendering software packages are used to reconstruct $3 \mathrm{D}$ views of the sample and to prepare animations.

The phase retrieval step can be very important, as is shown in Fig. 1. This figure shows part of a fly's leg. Phase contrast is clearly visible as the bright fringe at edges. These fringes produce artifacts in the reconstructed slices as is clearly seen in the slice reconstructed from the original data. The slice reconstructed from phase retrieved images has lower noise and significantly reduced level of artifacts.

The XuM microtomography system has been used to study a wide range of materials science, biological and industrial samples, see for example Fig. 2 which shows 3D rendered images of a European wasp foot, a jarrah wood sample and punctures in rubber. 


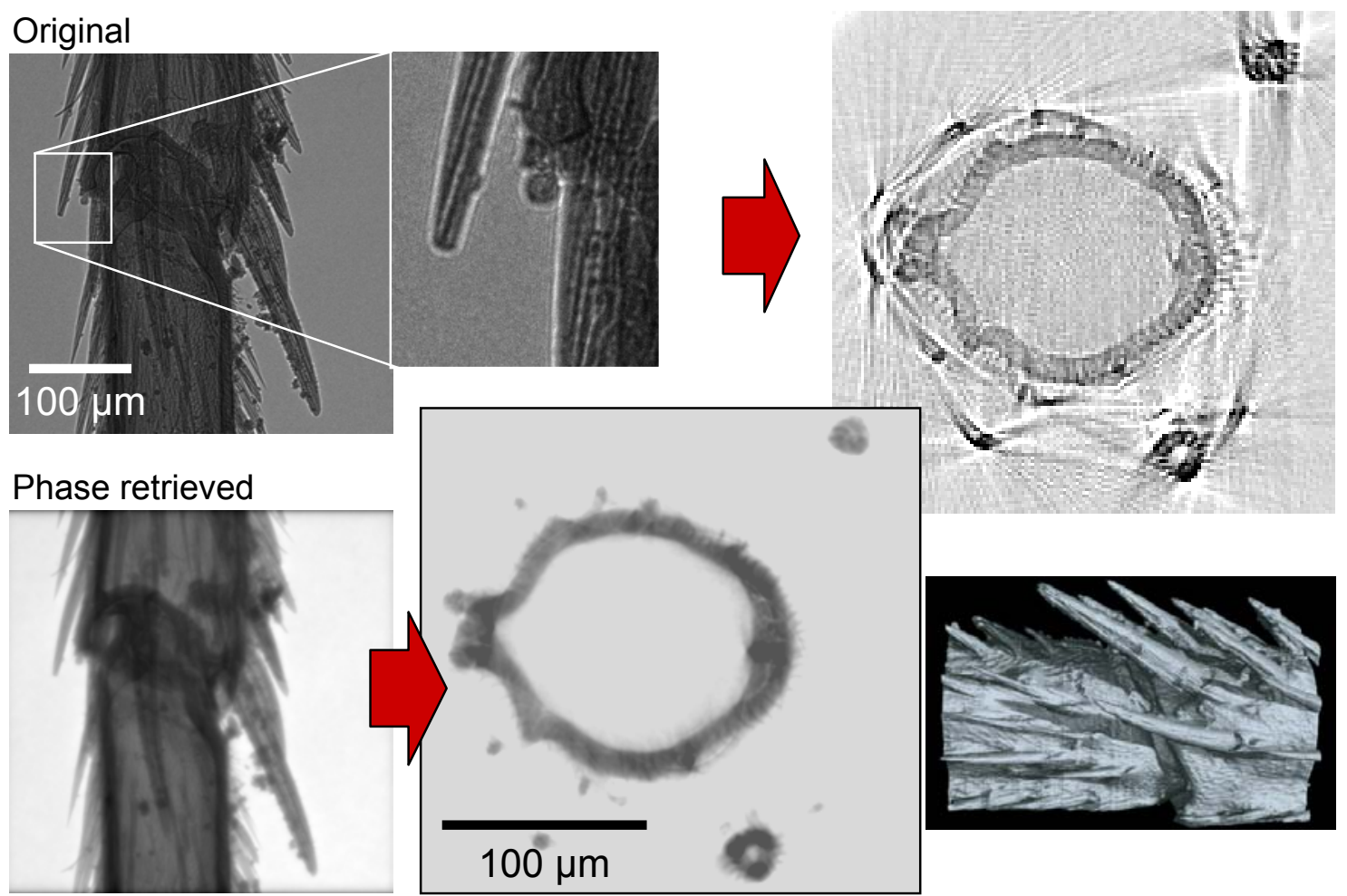

Fig. 1. Fly's leg sample. Comparison of reconstructed cross-section using original data and phase retrieved data.
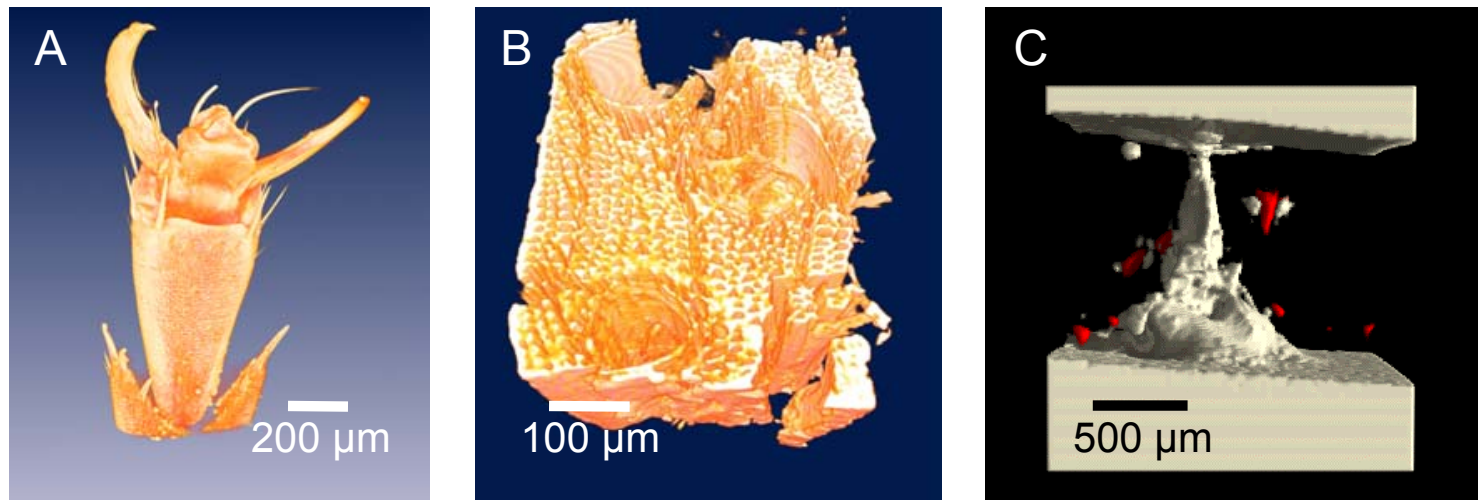

Fig. 2. Tomography examples: A) European wasp foot, B) jarrah wood and C) punctures in rubber.

References

[1] V.E. Cosslett and W.C. Nixon, J. App. Physics V24 Number 5, pp 616-623 (1953)

[2] S.C Mayo, P.R. Miller, S.W. Wilkins, T.J. Davis, D. Gao, T.E. Gureyev, D. Paganin, D.J. Parry, A. Pogany, and A.W. Stevenson, J. Micros., 207, pp 79-96 (2002).

[3] S.C. Mayo, T.J. Davis, T.E. Gureyev, P.R. Miller, D. Paganin, A. Pogany, A.W. Stevenson, S.W. Wilkins, Optics Express, 11, pp 2289-2302 (2003)

[4] L.A. Feldkamp, L.C. Davis and J.W. Kress, J.Opt.Soc.Am. A1, pp 612-619 (1984). 